\title{
Attribution Analysis on Brands Choice of Liquor in Symbolic Consumption
}

\author{
Ji Zeng \\ Sichuan University of Science and Engineering, Zigong, 643000, China
}

Keywords: Symbolic consumption. Brands. Attribution analysis

\begin{abstract}
This paper based on attribution analysis, researched the impact of liquor brands choice in symbolic consumption. The research found that the in the brands choice of liquor and others symbolic consumption, the external environment attribution of social relationship and personal attribution of consumer preference play significant role, and the external environment influenced consumers' brands choice greatly. The symbolic consumption of liquor and others emphasized on the intrinsic value realization on social, relationship, self-value, etc. Analyzing the decision behavior of symbolic consumption by the attribution theory of environmental and personal attribution has a direct revelation on liquor's brands competition and brands building.
\end{abstract}

\section{Literature review}

\section{The development of attribution theory}

Attribution theory is an important part of social psychology, which focuses on the process that people analyzing the behavior of themselves or others, judging behavior nature and cause. Fritz Heider firstly proposed the attribution theory in his work Interpersonal Relation Psychology (1958). Edward E. Jones and Keith Davis proposed the inference theory to infer behavior intention from the specific behavior of the actor. Harold H. Kelley proposed Three-dimensional Theory in Attribution Theory of Social Psychology and thought that the actors, actors' opponents and behavioral environment are three different kinds of explanations. Liu Yongfang introduced the cognitive process of attribution, attribution effects and the application of attribution theory in his Attribution Theory and Its Applications (The first edition of 2010). This book sorted and compared a variety of theories and models emerged in attribution studies, and clarified the attribution theory and the context of model development. Many domestic scholars also made studies on attribution theory from various perspectives of customer satisfaction, consumer behavior, enterprise social responsibility, etc.

\section{Study on symbolic consumption}

Symbolic consumption is widespread in social life, which does not emphasize on the functional properties of consumer goods, but more emphasize on internal relationships and inherent selfmanifestation, and liquor consumption is typical symbolic consumption. Bourdieu and Busia believed that consumption possesses symbolic meaning, which can distinguish social status, class brands and life style. Qi Haifeng, from the East China University of Technology proposed that symbolic consumption is an important means for individuals in the modern society in obtaining a sense of presence. Cui Nan, Wang Changzheng, from the Wuhan University summarized four dimensions, including personal, social, relation and collective dimensions, and proposed a symbolic consumption measurement table for measuring different brands.

The basic points derived from the above researches on symbolic consumption are "the demands and motivations of symbolic consumption are derived from the consumers' ego", which reflected the personal attribution of attribution theory. This paper based on the attribution theory, analyzed the consumption of liquor, and found that the liquor consumption is more prominent in external environment attribution of "social ego," "relationship ego ", etc. In liquor consumption, different environmental attributions of identity, ritual practices tendencies has a significant impact on the choice of specific liquor brands 


\section{The concept of the impact of symbolic consumption on brands choice}

The brands choice in the process of symbolic consumption of liquor is actually the consumer's ego-realization and peak experience, and is their preference (identity and preference degree) on brands reflected in the three aspects of "object (functional property of the consumer goods)", "relationship" (external environment) and "ego" (personal preference).

attribution theory proposes that after consumption, the consumers will produce "counterfactual", which is a kind of psychic reaction as people made wrong decisions (Bell, 1982), i.e. "regret". The measurement of "regret" degree is one of the tools in analyzing, judging and deciding the customer behavior through attribution theory.

Hoyerand Brown (1990) thought that before purchasing, most of the consumers have established specific tastes and preferences. Optimum brand is the consumers' most favorite brand, which best meet the social image, the relationship image, collective image and personal image of the specific consumer environment among environmental attributions. Sub-optimum is the "cost performance" option the consumers made according to quality, budget, etc. Most of the optimum brands are renowned expensive brands and sub-optimum brands are semi- known brands with stable quality and affordable price.

\section{Model analysis}

$$
\mathrm{Si}=\mathrm{Ui} \pm \mathrm{Bi} \pm \mathrm{Ki}, \quad \mathrm{i}=\mathrm{m}, \mathrm{n}
$$

Among which, Si refers to regret value. m, optimum brand. n, sub-optimumbrand. Ui, upward counterfactual, $\mathrm{Ui} \geq 0$; $\mathrm{Bi}$ brand culture attribution, $\mathrm{Bi} \geq 0$. Ki, consumer attribution, $\mathrm{Ki} \geq 0$. Vi, actual value. Di, desired value. As Vi-Di $\geq 0$, i.e. as the result of the choice behavior is positive, the impact of corresponding attribution on regret value $\mathrm{S}$ is reduced, the symbol " \pm " is " - ". As $\mathrm{Vi}-\mathrm{Di}<0$, the impact is increased, i.e. the result of the choice behavior is negative, the symbol " \pm " is " + ".

Hypothesis 1: select optimum and sub-optimum brands, the consumption results are recognized.

(1). According to the hypothesis, the results of the choice behavior is positive, i.e. $\mathrm{Vi}-\mathrm{Di} \geq 0$, $\mathrm{i}=\mathrm{m}, \mathrm{n}$, thus the symbol " \pm " in the formula is “-", i.e. $\mathrm{Sm}=\mathrm{Um}-\mathrm{Bm}-\mathrm{Km}, \mathrm{Sn}=\mathrm{Un}-\mathrm{Bn}-\mathrm{Kn}$.

(2). The attribution theory proposes that no natter the consumers choose the optimum brand or the sub-optimum brand, they will have certain "regret emotion", i.e. upward counterfactual that has upward influence to regret value S (U). Due to the differences between "optimum" and "sub-optimum" in brands, as consumers choose the sub-optimum brand, they will produce predicted results thinking about this choice and also concerns about the results of choosing the optimum brand, i.e. consumers will produce more upward counterfactual (U) as choosing sub-optimum brand than that of optimum brand, i.e.: upward counterfactual attribution $\mathrm{Um}<\mathrm{Un}$.

(3). The attribution theory proposes that as the behavior consequence happened, if the consequence is positive judgment, the actor will attribute more results and motivations on himself to improve their image recognition. While, is the consequence is negative judgment, the actor will attribute more results and motivations on external factors or environmental factors to achieve the purpose of self-protection.

In this hypothesis, if the both are positive results, the consumers have positive attributions on the brands, but in the general situation, there is "cost performance" factor that the consumers choose sub-optimum brand, thus the attribution on optimum brand is greater, i.e.: brand attribution: $\mathrm{Bm}>$ Bn.

(4). In this hypothesis, consumer will attribute the good results to their own judgment as choosing sub-optimum brand achieved the same satisfaction, i.e. consumer attribution $\mathrm{Km} \leq \mathrm{Kn}$.

(5). As the attribution on sub-optimum brand inevitably has the factor of "cost performance", it can be considered that consumer attribution $\mathrm{Ki}$ and brand attribution $\mathrm{Bi}$, has slighter impact on $\mathrm{S}$, the "regret value", i.e. $(\mathrm{Bm}-\mathrm{Bn})>(\mathrm{Kn}-\mathrm{Km})$. 
In this hypothesis, analyzing by combining various points, the formula is transformed as:

$\mathrm{Sm}=\mathrm{Um}-\mathrm{Bm}-\mathrm{Km}$

$\mathrm{Sn}=\mathrm{Un}-\mathrm{Bn}-\mathrm{Kn}$

Among which, $\mathrm{Um}<\mathrm{Un}, \mathrm{Bm}>\mathrm{Bn}, \mathrm{Km} \leq \mathrm{Kn}, \quad(\mathrm{Bm}-\mathrm{Bn})>(\mathrm{Kn}-\mathrm{Km})$

Hereby, $\mathrm{Sm}<\mathrm{Sn}$ 。

Hypothesis 2, the selections of optimum brand and sub-optimum brand are negative.

(1). According to the hypothesis, the results of the choice behavior are negative, i.e. $\mathrm{Vm}-\mathrm{Dm}<0$, the symbol " \pm " is "+", i.e.:

$\mathrm{Sm}=\mathrm{Um}+\mathrm{Bm}+\mathrm{Km}, \quad \mathrm{Sn}=\mathrm{Un}+\mathrm{Bn}+\mathrm{Kn}$

(2). Upward counterfactual based on the attribution theory thought that the hypothetical situation in a normal state, and the attribution is $\mathrm{Um}<\mathrm{Un}$.

(3). In this hypothesis, as produced negative result, people often attribute this result to external factors or environmental factors, i.e. attributed to the brands. Due to the differences between "optimum" and "sub-optimum" in brands, consumers will attribute more to sub-optimum brand, i.e.: brand attribution $\mathrm{Bm}<\mathrm{Bn}$.

(4). As the consumers attribute more negative results on external factors or environmental factors, thus in t his condition, it can be considered that consumers' attribution on personal factors is at an approximately low level.

In this hypothesis, analyzing by combining various points, the formula is transformed as:

$\mathrm{Sm}=\mathrm{Um}-\mathrm{Bm}-\mathrm{Km}$

$\mathrm{Sn}=\mathrm{Un}+\mathrm{Bn}+\mathrm{Kn}$

Among which, $\mathrm{Um}<\mathrm{Un}, \mathrm{Bm}<\mathrm{Bn}, \mathrm{Km}=\mathrm{Kn}$

Hereby, $\mathrm{Sm}<\mathrm{Sn}$

Hypothesis 3, the result of choosing optimum brand is positive while that of the sub-optimum brand is negative.

(1). According to the hypothesis, the result of choosing optimum brand is positive, i.e. Vm-Dm $\geq 0$, and the symbol " \pm " in the formula is " $-"$. While the result of choosing sub-optimum brand is negative, i.e. $\mathrm{Vm}-\mathrm{Dm}<0$, and the symbol " \pm " in the formula is " + ".

That is: $\mathrm{Sm}=\mathrm{Um}-\mathrm{Bm}-\mathrm{Km}, \mathrm{Sn}=\mathrm{Un}+\mathrm{Bn}+\mathrm{Kn}$

(2). Upward counterfactual based on the attribution theory thought that the hypothetical situation is that the "optimum brand" is superior to "sub-optimum brand", and in general situation, the attribution is $\mathrm{Um}<\mathrm{Un}$.

(3). In this hypothesis, consumers will produce "because I did well" for the optimum brand, while produce "because the brand is not good" for the sub-optimum brand, which resulted in observe from the perspective of personal attribution. The proportion of these two situations are fair, i.e. consumer attribution $\mathrm{Km}=\mathrm{Kn}$.

In this hypothesis, analyzing by combining various points, the formula is transformed as:

$\mathrm{Sm}=\mathrm{Um}-\mathrm{Bm}-\mathrm{Km}$

$\mathrm{Sn}=\mathrm{Un}+\mathrm{Bn}+\mathrm{Kn}$

Among which, $\mathrm{Um}<\mathrm{Un}, \mathrm{Bm}<\mathrm{Bn}, \mathrm{Km}=\mathrm{Kn}$

Hereby, $\mathrm{Sm}<\mathrm{Sn}$

Hypothesis 4, the result of choosing optimum brand is negative while that of the sub-optimum brand is positive

(1). According to the hypothesis, the result of choosing optimum brand is negative, i.e. Vm-Dm $<$ 0 and the symbol of " \pm " in the formula is "+". While the result of choosing sub-optimum brand is positive, i.e. $V m-D m \geq 0$, and the symbol of " \pm " in the formula is "-".

That is: $\mathrm{Sm}=\mathrm{Um}+\mathrm{Bm}+\mathrm{Km}, \mathrm{Sn}=\mathrm{Un}-\mathrm{Bn}-\mathrm{Kn}$

(2). In this hypothesis, as the result of choosing optimum brand is negative, consumers will produce more upward counterfactual (U), thus it can be considered that the counterfactual on optimum brand and sub-optimum brand are approximate, i.e. $\mathrm{Um}=\mathrm{Un}$. 
(3). Although the results violated consumer expectations, due to the differences between optimum and sub-optimum brads, consumers are rarely prone to attribute the negative results to optimum brand, although they do not attribute more to the sub-optimum brand, i.e. brand attribution $\mathrm{Bm}<\mathrm{Bn}$.

(4). In this hypothesis, consumers' choice behaviors are failed, but they are prone to emphasize on the successful personal attribution and reduce negative personal attribution, i.e. $\mathrm{Km}<\mathrm{Kn}$

In this hypothesis, analyzing by combining various points, the formula is transformed as:

$\mathrm{Sm}=\mathrm{Um}+\mathrm{Bm}+\mathrm{Km}, \mathrm{Sn}=\mathrm{Un}-\mathrm{Bn}-\mathrm{Kn}$

Among which, $\mathrm{Um}=\mathrm{Un}, \mathrm{Bm}<\mathrm{Bn}, \mathrm{Km}<\mathrm{Kn}$

Hereby, $\mathrm{Sm}>\mathrm{Sn}$

\section{Analysis conclusion of model}

Through the above model analysis, among the four hypotheses, only as the result of choosing optimum brand is negative, while the result of choosing sub-optimum brand is positive, the consumers will regret choosing the optimum brand. Under other three situations, consumers are prone to regret choosing the sub-optimum brand. Through the analysis, we can conclude that environmental and personal attributions are favor of optimum brand. Attribution theory proposed that this is because consumer behaviors are measured from personal, social, relationship and collective dimensions, and consumers are prone to emphasize on the interpersonal social relations constituted by relationships between people. Meanwhile, personal consciousness is an important attribution in analyzing, judging and making decision.

\section{Revelation on brand competition and brand establishment of liquor}

According to the above analysis, as consumers made choice on liquor brands, the sub-optimum brands are more likely to produce negative attribution. The sub-optimum brands of symbolic consumption of liquor should follow the following two points in the competition: firstly, enhancing the advantage of "cost performance", i.e. emphasize on the brands quality, build solid product functionality, strictly control the sensory quality, alcoholic strength, solid content, hygienic index, etc. of liquor. Secondly, establishing distinctive brand advantages, i.e. establishing strong brand culture from identity tendency (successful identity, style identity, taste identity, popular identity) and ritual practices tendencies (such as, a typical wedding etiquette) and targeting market segments of various social relations to focus on marketing.

\section{References}

[1] Bobby J. Calder, Robert E. Burnkrant, 1997 "Interpersonal Influence on Consumer Behavior: An Attribution Theory Approach", Journal of Consumer Research, Vol.4.

[2] Donovan, Robert, John, Marcoolynn, Nesdale. Store atmosphere and urchasing behavior. Journal of Retailing,1994,70(3):283-294

[3] LICHTENSTEIN D R, DRUMWRIGHT M E,BRAIG B M.The Effect of Corporate Social Respon-sibility on Customer Donations to Corporate-Sup-ported Nonprofits. Journal of Marketing, 2004,68(4):16 32.

[4] TSAI S P. Impact of personal orientation on luxurybrand purchase value.International Journal of Market Research,2005,47(4):429-450.

[5] O.CASS A.A psychometric evaluation of a revised version of the Lennox and Wolfe revise self-monitoring scale.Psychology \& Marketing ,2000,5(17):419-421.

[6] HSIEHM H,PAN S L, SETIONO R. Product-,Corporate-, and Country-Image Dimensions and Purchase Behavior: AMulticountry Analysis. Journal of theAcademy ofMarketing Science, 2004, 32(3): 251-270.

[7] THOMPSON C J, ARNOULD E J. Consumer Culture Theory(CCT): Twenty Years of Research. Journal of Consumer Research, 2005, 31(4): 868-882. 
[8] Zhang Wenhui, etc. The Impact of the Cognitive Characteristics of Decision Makers on Decision-Making Process and Enterprises' Strategic Choice. Acta Psychologica Sinica, 2005, 37 (3): 373-381

[9] Liu Yongfang. Attribution Theory and Its Application. Jinan City: Shandong People's Publishing House, 1998

[10] Chang Yaping, Yan Jun, Fang Qi. The Influence of Enterprise Social Responsibility Behavior and Product Prices on Consumer's Purchase Intentions. Journal of Management, 2008,5(1):110 117.

[11] Luli , Fan Xiucheng, Zheng Yuxiang. Marketing Theory of Conspicuous Consumption. Economic Management,2006,(15):54-56.

[12] Jiang Yan, Han Qiuyue. Comment on Conspicuous Consumption in Marketing Scenario. Hubei University of Economics Academic Journal, 2007,5 (4):71-75.

[13]Qi Haifeng. Analysis on Existential Psychology of Symbolic Consumption Behavior. East China Economic Management, 2007.4(21):101-104.

[14] Yan Zigang, Wang Xiuqiong. Shaping the Brand Personality on the Basis of Consumer Acceptance. Market Modernization, 2007(7):120-121

[15] Ju Huibing. Commodity symbolization: from use value to symbol value. Academic Journal of Beijing College of Commerce, 2000, (1). 